\title{
A Fast Convex Optimization Approach to Segmenting 3D Scar Tissue from Delayed-Enhancement Cardiac MR Images
}

\author{
Martin Rajchl ${ }^{1,2, \star}$, Jing Yuan ${ }^{1}$, James A. White ${ }^{1,3}$, Cyrus M.S. Nambakhsh ${ }^{1,2}$, \\ Eranga Ukwatta ${ }^{1,2}$, Feng Li ${ }^{1,2}$, John Stirrat ${ }^{1}$, and Terry M. Peters ${ }^{1,2}$ \\ 1 Imaging Laboratories, Robarts Research Institute, London, ON \\ 2 Department of Biomedical Engineering, Western University, London, ON \\ 3 Division of Cardiology, Department of Medicine, Western, University, London, ON \\ mrajchl@robarts.ca
}

\begin{abstract}
We propose a novel multi-region segmentation approach through a partially-ordered Potts (POP) model to segment myocardial scar tissue solely from 3D cardiac delayed-enhancement MR images (DEMRI). The algorithm makes use of prior knowledge of anatomical spatial consistency and employs customized label ordering to constrain the segmentation without prior knowledge of geometric representation. The proposed method eliminates the need for regional constraint segmentations, thus reduces processing time and potential sources of error. We solve the proposed optimization problem by means of convex relaxation and introduce its duality: the hierarchical continuous max-flow (HMF) model, which amounts to an efficient numerical solver to the resulting convex optimization problem. Experiments are performed over ten DEMRI data sets. The results are compared to a FWHM (full-width at half-maximum) method and the inter- and intra-operator variabilities assessed.
\end{abstract}

Keywords: Image segmentation, DE-MRI, Convex relaxation.

\section{Introduction}

Clinical interest in myocardial scar imaging using delayed enhancement magnetic resonance imaging (DE-MRI) has expanded over the past decade. A potential for DE-MRI to guide cardiovascular procedures, such as ablative therapies for elimination of atrial or ventricular arrhythmias and the optimal placement of pacemaker leads to treat heart failure (Cardiac Resynchronization Therapy), is now being appreciated [12. However, ability to translate this information into the procedural environment is a significant challenge. The recent validation of high-resolution isotropic 3D DE-MRI techniques provides superior spatial characterization of scar compared to its 2D predecessor 1 1 . Further, this dataset provides an unprecedented capacity to accurately represent scar within volumetric models to guide cardiac intervention. However, the efficient and accurate

\footnotetext{
^ Corresponding author.
} 
segmentation of scar signal from these datasets presents a significant challenge. Recently, several approaches 23] were proposed to segment the 3D scar tissue, which employ additional information from other images to constrain the search for scar tissue within the myocardium geometrically. Additional myocardial segmentations [4, or registrations [5] to other images is time consuming and a potential source of error.

Contributions. In this study, we propose a novel multi-region segmentation method to extract myocardial 3D scar tissue from high-resolution DE-MRI volume data sets. For this purpose, we introduce a POP model which uses prior knowledge of the anatomical spatial consistency of cardiac structures as additional constraints, rather than geometry. In particular, we solve the formulated non-convex optimization problem in terms of convex relaxation, by proposing a new $H M F$ formulation and demonstrate its duality to the convex relaxed POP model. We show that such a convex HMF model allows for a fast algorithm in modern convex optimization theory, which can be implemented on parallel computation platforms to reduce processing time using commercially available graphics hardware. The technique was tested using 3D DE-MRI datasets $(\mathrm{N}=10)$ obtained at 3 Tesla in patients with prior myocardial infarction.

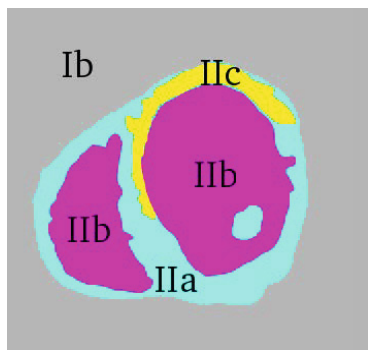

(a)

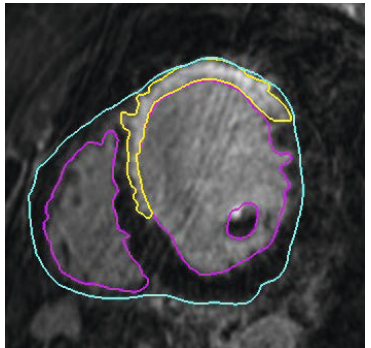

(b)

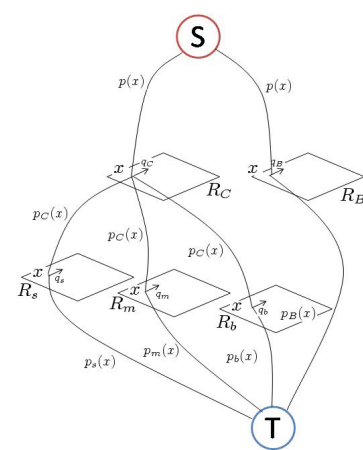

(c)

Fig. 1. Proposed label ordering based on anatomic spatial consistency (a) and contours overlaid on a DE-MRI slice (b). The region constraining the heart Ia) is split up in three subregions: IIa) myocardium, IIb) blood and IIc) scar tissue. Ib) represents the thoracic background. The graph in 1(c) shows the respective regions and flow configuration.

\section{Methods}

In the DE-MRI volume, we can clearly identify several compartments (see Fig. $1(\mathrm{a}) \mathrm{D}$ : the cardiac region and $\mathrm{Ib}$ ) thoracic background, where the cardiac compartment further contains three spatially coherent sub-regions: IIa) myocardium, IIb) blood volume and IIc) scar tissue; each of the three cardiac sub-regions has its distinct appearance model, which constitutes the complex appearance model 
of cardiac anatomy in DE-MRI. In this paper, we employ such a complex appearance model to assist segmenting the cardiac region accurately, which, in turn, automatically helps to identify the inherent sub-region of scar tissue. In fact, 6] shows the application of such complex appearance model significantly improves the segmentation accuracy for imaging which can not be simply modeled by independent and identically distributed random variables. We introduce the POP model to multi-region cardiac segmentation, which properly encodes prior information of label order.

\subsection{Partially-Ordered Potts Model and Convex Relaxation}

We segment the given DE-MRI volume $\Omega$ into multiple regions such that

$$
\Omega=\Omega_{C} \cup \Omega_{B}
$$

and

$$
\Omega_{C}=\Omega_{s} \cup \Omega_{m} \cup \Omega_{b}
$$

where $\Omega_{C}$ and $\Omega_{B}$ represent the two disjoint regions: Ia) the cardiac region and Ib) the thorical background; $\Omega_{s, m, b}$ represent the sub-regions of scar tissue, myocardium and blood respectively, which are disjoint from each other. To simplify our notations, we define the label sets: $L=\{C, B\}$ and $C=\{s, m, b\}$. Clearly, (2) states a partial order of regions, which can be incorporated into Potts model such that:

$$
\min _{\Omega_{C}, B, \Omega_{s, m, b}} \sum_{l \in L \cup C} \int_{\Omega_{l}} \rho(l, x) d x+\sum_{l \in L \cup C}\left|\partial \Omega_{l}\right|
$$

subject to the region constraints (11) and (2), where $\rho(l, x)$ gives the cost to label the pixel $x$ by $l \in L \cup C$ and $\left|\partial \Omega_{l}\right|$ represents the weighted length of the region $\Omega_{l}$. In practice, the cost for labeling the cardiac region can be fixed to 0 , i.e. $\rho(C, x)=0$; such that the cost for the cardiac region equals to the total cost of its contained three sub-regions: scar tissue, myocardium and blood. In this paper, we call (3) the POP model. Let $u_{l}(x) \in\{0,1\}, l \in L \cup C$, be the indicator function of the corresponding region $\Omega_{l}$ and $\omega_{l}(x)$ its regularization weight. Then the POP model (3) can be rewritten as

$$
\min _{u_{l \in L \cup C}} \sum_{l \in L \cup C} \int_{\Omega} u_{l}(x) \rho(l, x) d x+\sum_{l \in L \cup C} \int_{\Omega} \omega_{l}(x)\left|\nabla u_{l}\right| d x
$$

subject to the constraints of the labeling functions $u_{l}(x)$ such that

$$
u_{C}(x)+u_{B}(x)=1, \quad \sum_{l \in C} u_{l}(x)=u_{C}(x) ; \quad u_{l \in L \cup C}(x) \in\{0,1\}
$$

for each $\forall x \in \Omega$. Clearly, (5) just corresponds to (11) and (2). In this work, we solve the POP model (4) by its convex relaxation:

$$
\min _{u_{l} \in L \cup C} \sum_{l \in L \cup C} \int_{\Omega} u_{l}(x) \rho(l, x) d x+\sum_{l \in L \cup C} \int_{\Omega} \omega_{l}(x)\left|\nabla u_{l}\right| d x
$$


subject to the convex constraints

$$
u_{C}(x)+u_{B}(x)=1, \quad \sum_{l \in C} u_{l}(x)=u_{C}(x) ; \quad u_{l \in L \cup C}(x) \in[0,1] .
$$

The binary constraints for $u_{l}(x), l \in L \cup C$, in (5) are relaxed into their convex version in (7). The formulation (6), thereafter, boils down to a convex optimization problem, namely the convex relaxed POP model.

\subsection{Hierarchical Continuous Max-Flow Model}

We introduce a new continuous HMF model which is dual to the convex relaxed POP model. For this, we introduce the two-level flow-maximization configurations in a spatially continuous settings (see Fig. 1(c)]: in addition to the source and sink terminals $s$ and $t$, we put 2 copies $R_{C}$ and $R_{B}$ of the image domain $\Omega$ in parallel at the upper level; and put 3 copies $R_{l}, l \in C$ i.e. $\{s, m, b\}$, of $\Omega$ in parallel at the bottom level. We link $s$ to the same pixel $x$ at each upper-level domain $R_{C}$ and $R_{B}$ and define the unique flow $p_{s}(x)$ along the link from $s$ to $x$; link $x$ at $R_{C}$ to the same pixel $x$ at each domain of $R_{l}, l \in C$, and define the unique flow $p_{C}(x)$ along the links; link each pixel of $R_{B}$ and $R_{l}, l \in C$, to the sink terminal $t$, and define the flows $p_{B}(x)$ and $p_{l}(x), l \in C$. Additionally, the spatial flow fields $q_{l}(x), l \in L \cup C$, are defined within each domain $\Omega_{l}$.

Hierarchical Continuous Max-Flow Model. Based on the above settings, we set up the flow capacity and conservation conditions: for domains at the upper level, we define the flow capacity constraints:

$$
\left|q_{l}(x)\right| \leq \omega_{l}(x), \quad l=\{C, B\} ; \quad p_{B}(x) \leq \rho(B, x),
$$

and the flow conservation constraints, i.e. the flow residues $G_{l}(x)$ vanish:

$$
G_{l}(x):=\left(\operatorname{div} q_{l}-p_{s}+p_{l}\right)(x)=0, \quad l=\{C, B\} ;
$$

for the domains at the bottom level, we define the flow capacity constraints

$$
\left|q_{l}(x)\right| \leq \omega_{l}(x), \quad p_{l}(x) \leq \rho(l, x), \quad l=\{s, m, b\},
$$

and the flow conservation constraints, i.e. the flow residues $G_{l}(x)$ vanish:

$$
G_{l}(x):=\left(\operatorname{div} q_{l}-p_{C}+p_{l}\right)(x)=0, \quad l=\{s, m, b\} .
$$

We propose the continuous HMF model which maximizes the total flow streaming from the source $s$ to the sink $t$, i.e.

$$
\max _{p, q} \int_{\Omega} p_{s}(x) d x
$$

subject to the flow constraints (8), (9), (10) and (11). There is no constraint for the flow functions $p_{s}(x)$ and $p_{C}(x)$.

Following the same analytical steps as [7, we can prove the duality between the continuous HMF model (12) and the convex relaxed POP model (6), where the labeling functions $u_{l}(x), l \in L \cup C$, work as the optimum multipliers to the respective flow conservation constraints of (9) and (11). 


\subsection{Hierarchical Continuous Max-Flow Algorithm}

The continuous HMF model proposes a convex optimization problem with a linear energy function subject to the linear equality constraints (9) and (11), besides the constraints (8) and (10) on flow values, i.e. flow capacities. Thereafter, an efficient augmented Lagrangian based algorithm, namely the continuous HMF algorithm, can be derived, which iteratively optimizes the following augmented Lagrangian function:

$$
\max _{p, q} \min _{u} L_{c}(u ; p, q):=\int_{\Omega} p_{s} d x+\sum_{l \in L \cup C}\left\langle u_{l}, G_{l}\right\rangle-\frac{c}{2} \sum_{l \in L \cup C}\left\|G_{l}\right\|^{2}
$$

subject to the flow capacity constraints (8) and (10).

Similar as the continuous max-flow algorithm proposed in [877, the continuous HMF algorithm explores two sequential steps at each $k$-th iteration:

- Maximize the augmented Lagrangian function $L_{c}(u ; p, q)$ over the flow functions $p_{s}(x), p_{l}(x)$ and $q_{l}(x)$ where $l \in L \cup C$ subject to the flow capacity constraints (8) and (10).

- Update the labeling functions $u_{l}(x)$, where $l \in L \cup C$, by

$$
u_{l}^{k+1}=u_{l}^{k}-c G_{l}(x), \quad l \in L \cup C .
$$

To achieve computation efficiency, a one-step projected gradient strategy is applied to the maximization over each flow function, which shows a fast and steady convergence in practice.

\section{$3 \quad$ Experiments}

We developed a graphical user interface for the proposed method and implemented the optimization algorithm on a parallel computing architecture (CUDA, nVidia Corp., Santa Clara, CA.) for a significant increase in computation speed. The user can place seeds for regions on three orthogonal slice views corresponding to one of the labels shown in Fig 1(a) From all the seeded regions, we obtain a cost from each sample histogram with a maximum log-likelihood calculation [9] and add these costs as data fidelity terms $D(x)=-\log P\left(I(x) \mid l_{i}\right)$. Additionally, we use seeds as hard constraint costs. This approach provides the ability to correct for intensity inconsistencies, such as artifacts or uncertain regions. The label IIc) representing the scar tissue will be component-thresholded to all connected components containing seeds. This ensures that only marked tissue is classified as scar while other regions of fibrous tissue (for example from the mitral valvular apparatus) are excluded (see Fig. 2(b)). For both label groups I) and II), the total variation penalties $\alpha$ and $\beta$ (Eq. (6) $)$ were designed in the form $\alpha, \beta=\lambda_{1}+\lambda_{2} \exp \left(-\lambda_{3}|\nabla I(x)|^{2}\right) . \lambda_{1}$ and $\lambda_{2}$ may be varied by the user, and $\lambda_{3}$ was fixed to the value of 10 during these experiments. Using this interface, users were asked to segment 10 DE-MRI data sets of different levels of 


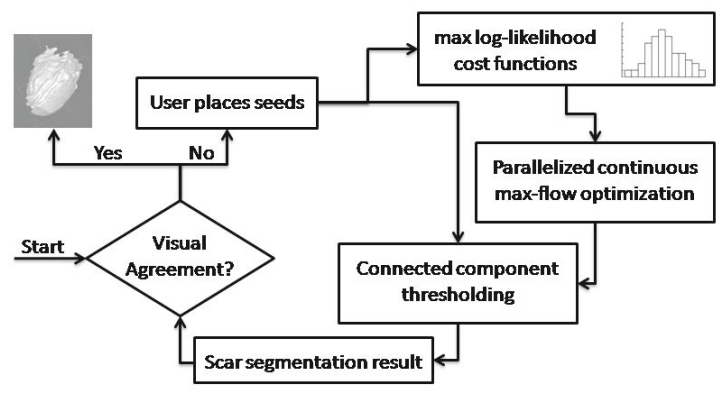

(a)

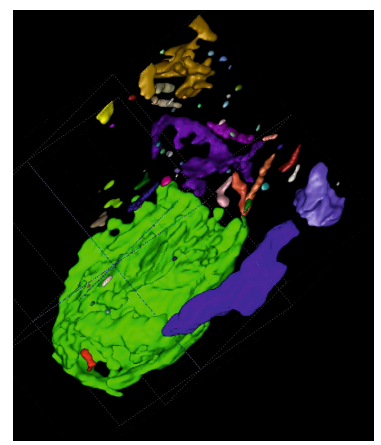

(b)

Fig. 2. (a) Proposed interactive segmentation pipeline. (b) Rendered example of intermediate segmentation result before connected component thresholding. While the image shows 38 separate components, only one of these components (green) represents scar tissue.

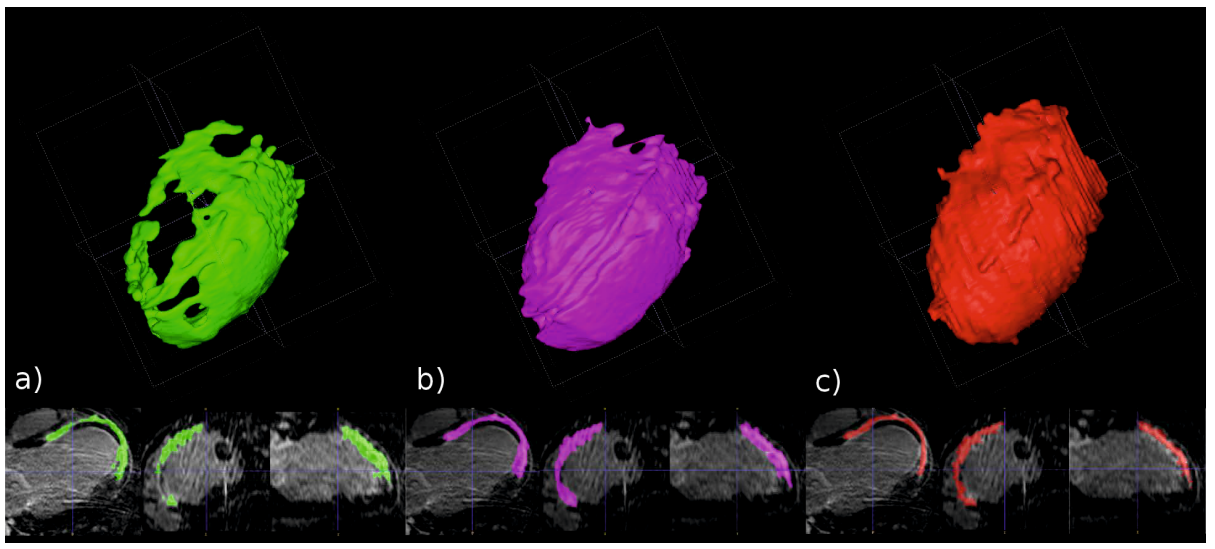

Fig. 3. Rendered example segmentation (top row) after a) the first and b) three recomputations. Rendered gold standard manual segmentation $c$ ) of the same scar tissue volume and respective slice views of segmentation on DE-MRI (bottom).

image quality containing scar volumes. The user places seeds for each label and computes the max-flow to obtain a segmentation result, and we record the time and the result, compared to a manually segmented ground truth of the scar.To assess robustness towards initialization and variability between operators, three users were asked to segment the same data set three times until they were satisfied with the result. Additionally we compared the segmentation results to those of the FWHM method. Constraining myocardial segmentations needed for the FWHM method were performed manually by a single expert and introduced a regional measure of overlap in form of a Dice coefficient (DSC) and a root mean squared surface error (RMSE) to measure the agreement of segmentation results to a single expert user manual segmentations. 


\section{Results}

Intermediate visual results of the segmentation pipeline after one and three interactions are shown in Fig. 3 .

Numerical results are shown in Table1. We observe an increase in mean overlap and at the same time a decrease in mean surface error as well as a decrease in their standard deviations. The proposed method outperforms a manually initialized FWHM method in every metric. Furthermore, the results of repeated segmentations $(\mathrm{N}=3)$ from three users are stated in Table 1 In this study, the users segmented the same randomly chosen images until they were satisfied with the results.

Table 1. Numerical results for proposed HMF method on data base $(\mathrm{N}=10)$ (left) and intra-/interoperator variabilities on the randomly-selected data set(right). A comparsion between FHWM and HMF-based segmentation is shown on the bottom.

\begin{tabular}{|c|c|c|c|c|c|}
\hline Interactions & $\operatorname{DSC}[0,1]$ & $\mathrm{RMSE}[\mathrm{mm}]$ & userID & $\operatorname{DSC}[0,1]$ & RMSE $[\mathrm{mm}]$ \\
\hline 1 & $0.55 \pm 0.17$ & $3.80 \pm 4.90$ & 1 & $0.77 \pm 0.02$ & $0.91 \pm 0.04$ \\
\hline 2 & $0.66 \pm 0.14$ & $3.03 \pm 4.90$ & 2 & $0.75 \pm 0.02$ & $1.05 \pm 0.18$ \\
\hline 3 & $0.69 \pm 0.14$ & $2.77 \pm 4.79$ & 3 & $0.74 \pm 0.01$ & $1.12 \pm 0.06$ \\
\hline 4 & $0.71 \pm 0.06$ & $1.44 \pm 0.62$ & & & \\
\hline 5 & $0.74 \pm 0.04$ & $1.40 \pm 0.62$ & All & $0.75 \pm 0.02$ & $1.02 \pm 0.14$ \\
\hline Method & $\operatorname{DSC}[0,1]$ & RMSE [mm] & \multicolumn{2}{|c|}{ Scar volume $[\mathrm{ml}]$} & volume error [\%] \\
\hline $\mathrm{HMF}$ & $0.78 \pm 0.03$ & $1.06 \pm 0.16$ & \multirow{2}{*}{\multicolumn{2}{|c|}{$\begin{array}{c}46.37 \pm 12.87 \\
14.42 \pm 4.73\end{array}$}} & $0.19 \pm 0.12$ \\
\hline FWHM & $0.47 \pm 0.10$ & $2.12 \pm 0.86$ & & & $-0.62 \pm 0.15$ \\
\hline
\end{tabular}

\section{Discussion and Conclusion}

We developed a segmentation framework based on a novel POP model approach to GPU-based convex relaxation, to segment 3D myocardial scar tissue from high-resolution DE-MRI volume data. Testing on 10 data sets of different quality showed that after a few interactions all error metrics decrease. The final segmentation results in the RMSE plus one standard deviation lie within the maximum image resolution of $1.3 \mathrm{~mm}$ and the user was able to get outliers from two data sets under control by correcting in critical regions. The performance of FWHM suggests that it is underestimating volumes from 3D DE-MRI. This might be due the increased variability of an intensity maximum occuring with increased sample size due to the additional spatial dimension. This might further lead to a threshold shift greatly underestimating scar extent. The GPU-based optimizer running on a Geforce 580 GTX provides high speed computations in less than 5 seconds. On average, it required $9 \pm 2$ minutes to extract the $3 \mathrm{D}$ scar volume from images. Inter- and intra-operator variability are both within $\pm 2 \%$ suggesting robustness towards initialization from different users. The observed performance and robustness suggests that the proposed segmentation method is 
suitable for clinical purposes, especially for the planning and guidance of interventional procedures reliant upon accurate spatial representation of myocardial scar tissue.

Acknowlegdements. We want to thank Dr. Aaron Ward and Eli Gibson for fruitful discussions and input on this topic. This project is supported by Canada Foundation for Innovation (CFI) grant \#20994, Canadian Institutes of Health Research (CIHR) grant MOP 179298 and ORF - RE-02-038. Martin Rajchl is enrolled in the CIHR Strategic Training Program in Vascular Research at Western University, London, ON.

\section{References}

1. White, J.A., Fine, N., Gula, L.J., Yee, R., Al-Admawi, M., Zhang, Q., Krahn, A., Skanes, A., MacDonald, A., Peters, T., Drangova, M.: Fused whole-heart coronary and myocardial scar imaging using 3-t cmr. implications for planning of cardiac resynchronization therapy and coronary revascularization. JACC. Cardiovascular Imaging 3(9), 921-930 (2010)

2. Andreu, D., Berruezo, A., Ortiz-Pérez, J., Silva, E., Mont, L., Borràs, R., de Caralt, T., Perea, R., Fernández-Armenta, J., Zeljko, H., Brugada, J.: Integration of 3d electroanatomic maps and magnetic resonance scar characterization into the navigation system to guide ventricular tachycardia ablation. Circulation. Arrhythmia and electrophysiology 4(5), 674-683 (2011)

3. Neizel, M., Boering, Y., Bönner, F., Balzer, J., Kelm, M., Sievers, B.: A fully automatic cardiac model with integrated scar tissue information for improved assessment of viability. Journal of Cardiovascular Magnetic Resonance 14(suppl. 1), M12 (2012)

4. Folkesson, F., Samset, E., Kwong, R., Westin, C.F.: Unifying statistical classification and geodesic active regions for segmentation of cardiac mri. IEEE Transactions on Information Technology in Biomedicine 12(3), 328-334 (2008)

5. Lehmann, H., Kneser, R., Neizel, M., Peters, J., Ecabert, O., Kühl, H., Kelm, M., Weese, J.: Integrating Viability Information into a Cardiac Model for Interventional Guidance. In: Ayache, N., Delingette, H., Sermesant, M. (eds.) FIMH 2009. LNCS, vol. 5528, pp. 312-320. Springer, Heidelberg (2009)

6. Delong, A., Gorelick, L., Schmidt, F.R., Veksler, O., Boykov, Y.: Interactive Segmentation with Super-Labels. In: Boykov, Y., Kahl, F., Lempitsky, V., Schmidt, F.R. (eds.) EMMCVPR 2011. LNCS, vol. 6819, pp. 147-162. Springer, Heidelberg (2011)

7. Yuan, J., Bae, E., Tai, X.-C., Boykov, Y.: A Continuous Max-Flow Approach to Potts Model. In: Daniilidis, K., Maragos, P., Paragios, N. (eds.) ECCV 2010, Part VI. LNCS, vol. 6316, pp. 379-392. Springer, Heidelberg (2010)

8. Yuan, J., Bae, E., Tai, X.: A study on continuous max-flow and min-cut approaches. In: CVPR 2010 (2010)

9. Boykov, Y., Jolly, M.: Interactive Graph Cuts for Optimal Boundary \& Region Segmentation of Objects in N-D Images. In: ICCV, pp. 105-111 (2001) 